\title{
Capacidade funcional em indivíduos com paraparesia espástica tropical/mielopatia associada ao HTLV-1
}

Functional capacity in individuals with tropical spastic paraparesis/HTLV-1-associated myelopathy

\section{Capacidad funcional en individuos con paraparesia espástica tropical/mielopatía asociada al HTLV-1}

\section{Recebido: 11/09/2017 \\ Aprovado: 21/12/2017 Publicado: 30/03/2018}

\author{
Victor Augusto Cavaleiro Corrêa ${ }^{1}$ \\ Ádria Cecília Botelho da Rocha ${ }^{2}$ \\ Priscila Sarmento de Almeida ${ }^{3}$ \\ Denise da Silva Pinto ${ }^{4}$ \\ Luiz Fábio Magno Falcão ${ }^{5}$ \\ Hellen Thaís Fuzii 6
}

Este estudo teve como objetivo avaliar a capacidade funcional de indivíduos com paraparesia espástica tropical/mielopatia associada ao vírus linfotrófico humano de células T do Tipo 1 (PET/MAH). A pesquisa incluiu 12 indivíduos diagnosticados com HTLV-1 de ambos os sexos, em acompanhamento no Laboratório de Estudos em Reabilitação Funcional (LAERF) da Universidade Federal do Pará (UFPA), através da aplicação do Health Assessment Questionnaire (HAQ). Este é um estudo quantitativo realizado em 2016. Dos participantes $58,3 \%$ eram do sexo feminino e $41,6 \%$ do sexo masculino. Apresentaram incapacidade moderada à grave $50 \%$ dos pesquisados, devido à dificuldade de deambular e alterações na postura $\mathrm{e}$ equilíbrio, necessitando assim de auxílio de terceiros e muletas e cadeiras de rodas. A problemática do viver com o HTLV-1 é abrangente e complexa, interferindo no cotidiano, estando relacionada a sintomatologia da doença e requerendo cuidados paliativos na capacidade funcional do dia-a-dia, principalmente, na funcionalidade que envolve os membros inferiores, as transferências de posturas e o equilíbrio.

Descritores: Paraparesia espástica tropical; Capacidade funcional; Vírus 1 linfotrópico T humano.

This study aimed to evaluate the functional capacity of individuals with tropical spastic paraparesis/myelopathy associated with human T-lymphotrophic virus type 1 (TSP/HAM). The study included 12 individuals diagnosed with HTLV-1 of both genders been treated in the Laboratory of Studies in Functional Rehabilitation (LAERF) of the Federal University of Pará (UFPA), Brazil, through the application of the Health Assessment Questionnaire (HAQ). This is a quantitative study conducted in 2016. Of the participants, $58.3 \%$ were female and $41.6 \%$ male. Fifty percent of those surveyed presented moderate to severe disability due to the difficulty of walking and changes in posture and balance, thus requiring help from third parties and crutches and wheelchairs. The issue of living with HTLV-1 is comprehensive and complex, interfering in the daily life, being related to the symptomatology of the disease and requiring palliative care in the day-to-day functional capacity, especially in the functionality involving lower limbs, transfers of postures and balance.

Descriptors: Paraparesis tropical spastic; Functional capacity; Human T - lymphotropic T vírus 1.

Este estudio tuvo como objetivo evaluar la capacidad funcional de individuos con paraparesia espástica tropical/mielopatía asociada al virus linfotrópico humano de células $\mathrm{T}$ del Tipo 1 (PET/MAH). La investigación incluyó 12 individuos diagnosticados con HTLV-1 de ambos sexos, en acompañamiento en el Laboratorio de Estudios en Rehabilitación Funcional (LAERF) de la Universidad Federal do Pará (UFPA), Brasil, a través de la aplicación del Health Assessment Questionnaire (HAQ). Este es un estudio cuantitativo realizado en 2016. De los participantes $58,3 \%$ eran del sexo femenino y $41,6 \%$ del sexo masculino. Presentaron incapacidad moderada a grave $50 \%$ de los investigados, debido a la dificultad de deambular y alteraciones en la postura y equilibrio, necesitando así de auxilio de terceros, muletas y sillas de ruedas. La problemática del vivir con el HTLV-1 es amplia y compleja, interfiriendo en lo cotidiano, estando relacionada a la sintomatología de la enfermedad y requiriendo cuidados paliativos en la capacidad funcional del día a día, principalmente, en la funcionalidad que implica los miembros inferiores, las transferencias de posturas y el equilibrio.

Descriptores: Paraparesia espástica tropical; Capacidad funcional;Vírus 1 linfotrópico T humano.

1. Terapeuta Ocupacional. Mestre em Psicologia. Doutor em Doenças Tropicais pela Universidade Federal do Pará (UFPA). Docente da Faculdade de Fisioterapia e Terapia Ocupacional (FFTO/UFPA), Belém, PA, Brasil. ORCID: 0000-0003-0133-7927 E-mail: victorcavaleiro@gmail.com

2. Terapeuta Ocupacional. Belém, PA, Brasil. ORCID: 0000-0001-7725-4496 E-mail: botelhodry@gmail.com

3. Terapeuta Ocupacional. Belém, PA, Brasil. ORDID: 0000-0003-4538-7934 E-mail: prideal07@gmail.com

4. Fisioterapeuta. Mestre em Epidemiologia. Doutora em Doenças Tropicais. Docente da FFTO, Belém, PA, Brasil. ORCID: 0000-0003-4940-8114

E-mail: denisepinto@ufpa.br

5. Fisioterapeuta. Mestre em Doenças Tropicais. Doutor em Virologia. Pesquisador da Universidade do Estado do Pará (UEPA). ORCID: 0000-0001-8391-2694 E-mail: fabiofalcao29@yahoo.com.br

6. Farmacêutica. Mestre em Microbiologia e Imunologia. Doutora em Pediatria e Ciências Aplicadas à Pediatria. Docente Associada da UFPA. ORCID: 0000-0002-0616-3543 E-mail: hellenfuzii@gmail.com 


\section{INTRODUÇÃO}

O vírus Linfotrófico Humano de Células T Tipo 1 (HTLV-1) é um vírus que infecta de 10 a 20 milhões de pessoas no mundo todo, considerado endêmico no Japão, Caribe, Melanésia, África, América Central e do Sul. No Brasil, há aproximadamente, 2,5 milhões de pessoas contaminadas no Brasil, com alta prevalência nas regiões Norte e Nordeste ${ }^{1,2}$.

O HTLV-1 foi o primeiro retrovírus humano descoberto e isolado, sendo relatado em uma clínica em 1977, no Japão. Foi descoberto também uma aglomeração de pacientes na parte sudoeste no Japão, e pesquisadores tiveram a hipótese de que a doença poderia ser induzida por um vírus ${ }^{3,4}$.

Existem 4 subtipos conhecidos do HTLV, sendo os mais importantes o HTLV-1 e 2, quanto a patogenia e epidemiologia. É transmitido através de relações sexuais, onde em pessoas do sexo feminino, a transmissão é quatro vezes maior, em comparação a transmissão da mulher para o homem pela via sexual, sanguínea e vertical $2,4,5$.

Outros meios de transmissão são pela utilização de produtos infectados com sangue do indivíduo com a doença, e transmissão vertical, a passada de mãe para filho, contudo aproximadamente $90 \%$ dos contaminados permanecerão assintomáticos, mantendo assim uma rede de transmissão silenciosa, contudo com baixa morbidade ${ }^{4,5}$.

0 HTLV-1 pode ser associado a várias patologias, entre elas, a Paraparesia Espástica Tropical/Mielopatia Associada ao HTLV (PET/MAH), uma manifestação neurológica, no qual a sua principal sintomatologia é déficit motor dos membros inferiores, afeta de $2 \%$ a $3 \%$ dos infectados ${ }^{4,6}$.

A PET/MAH é uma Mielopatia associada, evidenciada por quadros de paraparesia progressiva crônica e compressão dos membros inferiores, hérnias de disco, perda sensorial, incontinência urinária, impotência sexual, constipação, hipotensão ortostática, déficits de atenção, déficits visuais e alguns apresentam dor lombar, com irradiação para os membros inferiores. Pode apresentar também sinais piramidais com presença de sinal de Babinski ${ }^{4,7}$.

Diante das características da PET/MAH, surgem algumas alterações orgânicas e a perda da capacidade funcional (CF). A CF pode ser definida como o grau de preservação da habilidade em executar, de forma autônoma e independente, as atividades de vida diária (AVD) e as atividades instrumentais de vida diária (AIVD), dependentes de habilidades físicas, mentais e condicionantes ambientais, como barreiras físicas e limitações de tempo, estando em permanente cuidados paliativos ${ }^{8}$.

0 cuidado paliativo compreende uma abordagem de cuidado diferenciada, que se propõe a promover a qualidade do viver do paciente e de seus familiares, através da prevenção e tratamento adequados para amenizar o sofrimento, por meio do diagnóstico precoce, avaliação eficaz, observação para evitar problemas físicos, além de providenciar suporte psicossocial e espiritual. Refere-se aos cuidados paliativos como cuidados intensivos, que requerem profissionais especializados, com conhecimentos e habilidades individualizadas para cada cliente ${ }^{9-11}$.

A assistência paliativa não deve ser considerada como uma opção depois de um tratamento curativo ineficaz, mas sim um conjunto de cuidados prestados ao paciente desde o princípio da terapêutica em doenças crônicas, sendo considerada uma abordagem especializada para ajudar o indivíduo a viver melhor até o momento de sua morte ${ }^{12}$.

Nesse sentido, a integralidade do ser humano é colocada em evidência nos cuidados paliativos, de modo que, possibilitem a expressão dos pensamentos e sentimentos que envolvem sua finitude, na perspectiva de auxiliá-los para o encerramento do ciclo de suas existências.

É importante não apressar ou adiantar a morte, e sim afirmar a vida e entender que a morte é um processo natural. Com os cuidados paliativos devem-se tentar aliviar algias e outros sintomas que levam à angústia; agregar aos pacientes cuidados no aspecto psíquico e espiritual; dar suporte para as pessoas manterem-se ativas até a morte 
chegar, além de ajudar a família no apoio à compreensão da doença, ou mesmo no seu processo de luto ${ }^{13}$.

A CF é primordial para a promoção de saúde de indivíduos acometidos por doenças crônico-degenerativas como o PET/MAH, podendo fornecer dados que podem somar no diagnostico, tratamento, reabilitação e na prevenção de complicações secundárias ${ }^{14,15}$.

Portanto, ressalta-se a importância em averiguar este aspecto no público desta pesquisa, com a finalidade de eleger os principais aspectos funcionais que apresentem déficits nas atividades cotidianas e afetem as rotinas destes indivíduos, ocasionando na ruptura e modificação dos seus papéis ocupacionais anteriormente vividos. Neste sentido, este estudo teve como objetivo avaliar a capacidade funcional de indivíduos com paraparesia espástica tropical / mielopatia associada ao vírus linfotrófico humano de células T do Tipo 1 (PET/MAH).

\section{MÉTODO}

Trata-se de um estudo descritivo com abordagem quantitativa que é um método de pesquisa onde se obtém dados numéricos. Caracteriza-se, tanto na fase de coleta de dados quanto no seu tratamento, pela utilização de técnicas estatísticas ${ }^{16}$.

Esta investigação foi realizada no Laboratório de Estudos em Reabilitação Funcional (LAERF) da Faculdade de Fisioterapia e Terapia Ocupacional (FFTO) do Instituto de Ciências da Saúde (ICS) da Universidade Federal do Pará (UFPA) entre os anos de 2015 a 2016.

Participaram desta pesquisa 12 indivíduos diagnosticados com HTLV-1, que apresentavam PET/MAH e faziam acompanhamento no LAERF/UFPA. Como critérios de inclusão: pacientes de ambos os sexos, com o HTLV - 1, que apresentavam a PET/MAH, faziam acompanhamento no LAERF e que aceitaram participar da pesquisa. E como critérios de exclusão: pacientes que não apresentaram sintomas relacionados ao vírus, não faziam tratamento no LAERF ou que não aceitaram participar da pesquisa.
Conforme a Resolução 466/12 do Conselho Nacional de Saúde (Brasil, 2012), o projeto de pesquisa foi aprovado pelo Comitê de Ética em Pesquisa com seres humanos do Instituto de Ciências da Saúde da Universidade Federal do Pará - ICS/UFPA, com o parecer $\mathrm{n} \mathbf{0} \mathbf{1 . 4 2 6 . 2 2 7}$ de 26 de fevereiro de 2016. Os participantes manifestaram o aceite em participar da pesquisa através da assinatura do Termo de Consentimento Livre e Esclarecido (TCLE).

Utilizou-se o Health Assessment Questionnaire (HAQ), que consiste em um questionário geral traduzido para o português e validado no Brasill7, desenvolvido há, aproximadamente, 30 anos, onde avalia a capacidade funcional através de 20 atividades divididas em oito categorias: limpeza e higiene, mobilidade, comer, andar, higiene, alcance, de preensão e atividades comuns do cotidiano ${ }^{18,19}$.

0 questionário é auto avaliativo, onde a pessoa pontua cada atividade de 0 a 3: 0 pontos é sem dificuldade, 1 ponto é pouca dificuldade, 2 pontos significa muita dificuldade e 3 pontos, a pessoa não consegue realizar. Diante disso, se obtém a média de cada categoria, onde quanto mais alto for $o$ escore, mais afetada esta a capacidade funcional dessa pessoa. A avaliação funcional também permite ter uma noção não apenas da força física, mas também da destreza e estado cognitivo do individuo ${ }^{14,19}$.

A idade e tempo de tratamento foram expressos em média e desvio padrão. Os demais dados foram expressos da seguinte forma, $\left(\mathrm{n}^{\mathrm{o}}\right)$ : frequência absoluta. F (\%) frequência em porcentagem.

\section{RESULTADOS}

A Tabela 1 apresenta a distribuição demográfica dos pacientes participantes da pesquisa.Dos 12 pacientes entrevistados, $58,4 \%$ eram do sexo feminino e $41,6 \%$ do sexo masculino.A média de idade para mulheres foi de 55,7 anos, já para os homens de 49,6 anos.

A maioria dos indivíduos é de origem Paraense com $83,4 \%$, sendo os outros $16,6 \%$ divididos entre Ceará e Amapá. 
Tabela 1. Perfil epidemiológico de pacientes com HTLV-1 - PET/MAH. Belém/PA, 2015-2016.

\begin{tabular}{|c|c|c|c|}
\hline \multirow[b]{2}{*}{ Variáveis } & \multicolumn{3}{|c|}{$\begin{array}{c}\text { Pacientes } \\
\mathrm{N}=12\end{array}$} \\
\hline & $\mathbf{N}$ & F (\%) & Média \\
\hline \multicolumn{4}{|l|}{ Sexo } \\
\hline Feminino & 07 & $58,4 \%$ & \\
\hline Masculino & 05 & $41,6 \%$ & \\
\hline \multicolumn{4}{|l|}{ Idade } \\
\hline Mulheres & - & - & 55,7 (média) \\
\hline Homens & - & - & 49,6 (média) \\
\hline \multicolumn{4}{|l|}{ Estado Civil } \\
\hline Solteiro (a) & 03 & $25,0 \%$ & \\
\hline Casado (a) & 07 & $58,4 \% *$ & \\
\hline Separado (a) & 01 & $08,3 \%$ & \\
\hline Viúvo (a) & 01 & $08,3 \%$ & \\
\hline \multicolumn{4}{|l|}{ Escolaridade } \\
\hline$<9$ & 05 & $41,7 \%$ & \\
\hline$>9$ & 07 & $58,3 \%$ & \\
\hline \multicolumn{4}{|l|}{ Naturalidade } \\
\hline Pará & 10 & $83,4 \%$ & \\
\hline Ceará & 01 & $08,3 \%$ & \\
\hline Amapá & 01 & $08,3 \%$ & \\
\hline \multicolumn{4}{|l|}{ Orientação Religiosa } \\
\hline Católico & 05 & $41,7 \%$ & \\
\hline Evangélico & 07 & $58,3 \%$ & \\
\hline \multicolumn{4}{|l|}{ Profissão } \\
\hline Formal & 05 & $41,7 \%$ & \\
\hline Informal & 07 & $58,3 \%$ & \\
\hline \multicolumn{4}{|l|}{ Tempo de Tratamento } \\
\hline Meses & - & - & 41,5 (média) \\
\hline Anos & - & - & 03,5 (média) \\
\hline \multicolumn{4}{|c|}{ Acompanhamento Profissional } \\
\hline Médico & 11 & $91,7 \%$ & \\
\hline Fisioterapeuta & 11 & $91,6 \%$ & \\
\hline Terapeuta Ocupacional & 03 & $25,0 \%$ & \\
\hline
\end{tabular}

Em relação à situação conjugal, houve predomínio de indivíduos casados com $58,4 \%, 25 \%$ solteiros, $16,6 \%$ entre separado e viúvo.

O tempo de tratamento deu-se em média de 3,5 anos, o que demonstrou a manifestação tardia do vírus e da PET/MAH. A maioria descobriu a doença a partir da doação de sangue e/ou pela manifestação dos primeiros sintomas. Destaca-se também, que apenas $25 \%$ tem acompanhamento com terapeuta ocupacional.

$\mathrm{Na}$ capacidade funcional, $50 \%$ apresentam incapacidade moderada à grave, com dificuldade de deambular e alterações na postura e equilíbrio e, alguns precisam de auxílio de familiares, muletas e cadeira de rodas.
41,7\% dos usuários apresentaram "Dificuldade leve à moderada". Um único usuário apresentou "incapacidade grave ou muito grave", o qual era cadeirante, dependente dos familiares (Tabela 2).

Tabela 2. Capacidade funcional pelo Questionário de Avaliação de Saúde (HAQ), referidos pelos pacientes com HTLV/1 PET/MAH. Belém/PA, 2015-2016.

\begin{tabular}{lccc} 
& & \multicolumn{2}{c}{ PET/MAH } \\
\multicolumn{1}{c}{ Variáveis } & & $\boldsymbol{F}$ (no) & $\boldsymbol{F ~ ( \% ) ~}$ \\
\hline $\begin{array}{l}\text { Dificuldade leve } \\
\text { moderada }\end{array}$ & à & 05 & $41,7 \%$ \\
$\begin{array}{l}\text { Incapacidade moderada à } \\
\text { grave }\end{array}$ & 06 & $50,0 \%$ \\
$\begin{array}{l}\text { Incapacidade grave ou } \\
\text { muito grave }\end{array}$ & 01 & $08,3 \%$ \\
\hline
\end{tabular}


As limitações em realizar as atividades foram medidas pelo Questionário de Avaliação pela Saúde (HAQ), dividido em oito categorias (Tabela 3). Percebe-se de modo mais detalhado em quais atividades os entrevistados apresentam maiores dificuldades.

Percebe-se que a categoria Higiene foi a que teve pior pontuação, $41,6 \%$ não conseguem fazer, devido ao fato desta exigir mais de marcha, postura e equilíbrio, aspectos no qual são os mais afetados pela PET/MAH, por atingir mais significativamente os membros inferiores.

Em contrapartida, as subdivisões que necessitam mais dos membros superiores

Tabela 3. Capacidade funcional pelo HAQ, subdivididos nas 8 categorias de atividades funcionais referidos pelos pacientes com HTLV/1 - PET/MAH. Belém/PA, 2015-2016.

\begin{tabular}{|c|c|c|}
\hline \multirow[b]{2}{*}{ Variáveis } & \multicolumn{2}{|c|}{$\begin{array}{c}\text { PET/MAH } \\
\mathrm{N}=12\end{array}$} \\
\hline & $F\left(n^{o}\right)$ & $F(\%)$ \\
\hline \multicolumn{3}{|l|}{ Limpeza e Higiene } \\
\hline 0 (Sem dificuldade) & 05 & $41,8 \%$ \\
\hline 1 (Pouca dificuldade) & 05 & $41,6 \%$ \\
\hline 2 (Muita dificuldade) & 02 & $16,6 \%$ \\
\hline 3 (Não consegue) & - & - \\
\hline \multicolumn{3}{|l|}{ Mobilidade } \\
\hline 0 (Sem dificuldade) & 03 & $25,0 \%$ \\
\hline 1 (Pouca dificuldade) & 03 & $25,0 \%$ \\
\hline 2 (Muita dificuldade) & 03 & $25,0 \%$ \\
\hline 3 (Não consegue) & 03 & $25,0 \%$ \\
\hline \multicolumn{3}{|l|}{ Comer } \\
\hline 0 (Sem dificuldade) & 08 & $66,8 \%$ \\
\hline 1 (Pouca dificuldade) & 02 & $16,6 \%$ \\
\hline 2 (Muita dificuldade) & 01 & $08,3 \%$ \\
\hline 3 (Não consegue) & 01 & $08,3 \%$ \\
\hline \multicolumn{3}{|l|}{ Andar } \\
\hline 0 (Sem dificuldade) & 04 & $33,3 \%$ \\
\hline 1 (Pouca dificuldade) & 02 & $16,6 \%$ \\
\hline 2 (Muita dificuldade) & 03 & $25,1 \%$ \\
\hline 3 (Não consegue) & 03 & $25,0 \%$ \\
\hline \multicolumn{3}{|l|}{ Higiene } \\
\hline 0 (Sem dificuldade) & 03 & $25,0 \%$ \\
\hline 1 (Pouca dificuldade) & 01 & $08,3 \%$ \\
\hline 2 (Muita dificuldade) & 03 & $25,0 \%$ \\
\hline 3 (Não consegue) & 05 & $41,7 \%$ \\
\hline \multicolumn{3}{|l|}{ Alcance } \\
\hline 0 (Sem dificuldade) & 04 & $33,3 \%$ \\
\hline 1 (Pouca dificuldade) & 02 & $16,7 \%$ \\
\hline 2 (Muita dificuldade) & 04 & $33,3 \%$ \\
\hline 3 (Não consegue) & 02 & $16,7 \%$ \\
\hline \multicolumn{3}{|l|}{ De Preensão } \\
\hline 0 (Sem dificuldade) & 10 & $83,4 \%$ \\
\hline 1 (Pouca dificuldade) & 02 & $16,6 \%$ \\
\hline 2 (Muita dificuldade) & - & - \\
\hline 3 (Não consegue) & - & - \\
\hline \multicolumn{3}{|l|}{ Atividades comuns do cotidiano } \\
\hline 0 (Sem dificuldade) & 03 & $25,0 \%$ \\
\hline 1 (Pouca dificuldade) & 01 & $08,3 \%$ \\
\hline 2 (Muita dificuldade) & 05 & $41,7 \%$ \\
\hline 3 (Não consegue) & 03 & $25,0 \%$ \\
\hline
\end{tabular}




\section{DISCUSSÃO}

A predominância feminina é explicada, devido à transmissão do HTLV-1 ser mais eficiente do homem para a mulher, havendo duas vezes mais chances de contaminação. Assim, o sexo feminino é um fator de risco para a infecção do vírus ${ }^{4,20,21}$.

Estes dados podem ser explicados pela alta taxa de infecção no Brasil, especialmente, em áreas endêmicas como nas regiões Norte e Nordeste 2 .

É possível que a transmissão sexual ser uma importante via de infecção do HTLV-1. Mas também se faz questionar se o alto número de pessoas casadas infectadas pelo vírus seria devido a relações extraconjugais sem o uso de preservativos ${ }^{2} 22$.

Os resultados estão em consonância com outros estudos que afirmam que pessoas com mais de 10 anos de sintomatologia. Destaca-se também que, a maioria deambula apenas com auxílio de algum recurso ${ }^{6}$.

0 HAQ é um questionário autoaplicável, sendo um dos instrumentos mais citados e usados em patologias reumáticas. Em relação ao HTLV, não existe estudos que relatem a abordagem desse questionário com pessoas com HTLV no norte do Brasil, especificamente no Estado do Pará23.

Em segundo entra Mobilidade, Andar e Atividades Comuns do Cotidiano, com 25\% em cada um ${ }^{4}$.

Isto se deve ao fato da paraparesia não afetar a força nos braços, mesmo uma porcentagem desses pacientes podendo apresentar a hiperreflexia. Com isso, o desempenho nessas duas categorias continua satisfatório, assim como nas categorias Limpeza e Higiene e Alcance 4 .

Essa condição revela que esses indivíduos se encontram em cuidados paliativos, com destaque para as atividades do dia-a-dia. Esses cuidados têm despertado crescente interesse da sociedade, tendo em vista que tem aumentado o número de pessoas acometidas por doenças crônicas que ameaçam a vida, necessitando, então, de cuidados essenciais como assistência contínua e integral ${ }^{24}$.

Além da atenção integral ao paciente que se encontra em cuidados paliativos, é necessária também atenção diferenciada aos familiares e cuidadores. A Organização Mundial da Saúde considera como um dos principais objetivos dos cuidados paliativos, a atenção às necessidades e a qualidade de vida de pacientes, familiares e cuidadores em processo de adoecimento, e, para isto, determina que seja disponibilizada atenção especial $^{9}$.

Ressalta-se a importância do Terapeuta Ocupacional no tratamento destes indivíduos, tendo em vista as inúmeras demandas físicas, psíquicas, emocionais e sociais em decorrência da patologia, no qual este profissional pode ajudar a diminuir o impacto das manifestações dos sintomas, através de novas possiblidades ocupacionais.

A Terapia Ocupacional é um campo de conhecimento e atuação em saúde e educação, que busca prevenir e tratar dificuldades físicas e/ou psicossociais, visando a melhoria da qualidade do viver ocupacional do indivíduo. Utiliza-se de fazeres como elemento orientador na estruturação do processo terapêutico. É fundamental sua intervenção, pois é responsável por analisar e promover vida ocupacional na rotina do paciente nos mais diversos aspectos ${ }^{25,26}$.

\section{CONCLUSÃO}

A problemática do viver com o HTLV é abrangente e complexa, provocando mudanças muitas vezes radicais, intervindo no cotidiano das pessoas com a PET/MAH.

Como evidenciado no estudo, tais mudanças estão extremamente correlacionadas com a sintomatologia da doença e demandando cuidados especiais quanto às atividades do dia-a-dia, a capacidade funcional e as habilidades que exigem mais dos membros inferiores, transferência de postura e equilíbrio.

A partir dos resultados encontrados nesta pesquisa, propõe-se através da Terapia Ocupacional intervenções e estratégias, como adaptações ambientais, o uso de cadeira de rodas adequadas e treino de atividades de vida diária, favorecendo uma maior autonomia e independência e buscando melhora na qualidade do viver destes 
indivíduos.

Destaca-se ainda que este estudo é o primeiro utilizando o HAQ em pesquisa com indivíduos com PET/MAH, em especial na região Amazônica.

Diante dos resultados encontrados, confirmam-se a importância da produção científica, análise e discussão sobre o HTLV para o desenvolvimento de políticas e estratégias de saúde voltadas a esses indivíduos e aos profissionais de saúde que acompanham esses casos.

Nesse contexto, mostra-se relevante a atuação da Terapia Ocupacional no cuidado com este público, a fim de contribuir para a diminuição das dificuldades encontradas por estes diante da adaptação a nova rotina, a mudança de papéis ocupacionais, na descoberta e aprendizado de habilidades para uma vida com a máxima autonomia possível.

Entre as limitações desta pesquisa, destacam-se a não análise causal entre a $\mathrm{PET} / \mathrm{MAH}$ e a capacidade funcional, a pequena amostra do estudo, devido a dificuldades relacionadas ao diagnóstico de infecção pelo HTLV-1 e ao acesso desses indivíduos aos serviços de saúde.

Recomenda-se que a capacidade funcional possa ser também identificada e monitorada como mais uma repercussão da infecção pelo HTLV-1, podendo ser mais uma condição na prevenção e promoção a saúde desses indivíduos. Além disso, devido ao impacto na saúde, sugere-se que outros estudos possam examinar a presença dessas manifestações em grupos similares.

\section{REFERÊNCIAS}

1. Tang SW, Ducroux A, Jeang KT, Neuveut C. Impact of cellular autophagy on viruses: Insights from hepatitis B virus and human retroviruses. J Biomed Sci. 2012; 19(92):1-11.

2. Glória LM, Damasceno SA, Rodrigues LR, Santos MSB, Medeiros R, Dias GAS, Pinto DS. Perfil clínicoepidemiológico de pacientes infectados pelo HTLV-1 em Belém/Pará. Cad Saúde Coletiva. 2015; 23(2):157-62.

3. Iwanaga M, Watanabe T, Yamaguchi K. Adult Tcell leukemia: a review of epidemiological evidence. Front Microbiol. 2012; 3:1-13.

4. Ministério da Saúde (Br). Guia do manejo clínico da infecção pelo HTLV [Internet]. Brasília, FD; 2013 [citado em 23 jun 2017]. Disponível em: http://www.sierj.org.br/artigos/htlv_manual_fin al_pdf_25082.pdf.

5. Semeão LES, Roque DR, Francisco Sobrinho T, Costa CKF, Dodorico M, Yamaguchi MU. Soroprevalência do vírus linfotrópico de células $\mathrm{T}$ humanas (HTLV) entre doadores de sangue em hemocentros de Maringá-Paraná e Boa VistaRoraima. Epidemiol Serv Saúde. 2015; 24(3):5239.

6. Santos ACC, Soares DJ, Rivemales MCC. (Des)conhecimento, adoecimento e limitações impostas pelo HTLV: experiências de mulheres soropositivas. Cad Saúde Coletiva. 2017; 25(1):4550.

7. Trindade BC, Sorgi CA, Nicolete LDF, Malta TM, Pinto MT, Takayanagui OM, et al. Leukotrienes are up regulated and associated with Human TLymphotropic Virus Type 1 (HTLV-1) - associated neuroinflammatory disease. PLos ONE. 2012; 7(12):1-11.

8. Leandro LA. Fatores associados ao desempenho funcional de idosos portadores da Doença de Parkinson. [Dissertação]. Curitiba: Universidade Federal do Paraná; 2011.

9. World Health Organization. Câncer [Internet]. [Geneva]: WHO; 2017 [citado em 01 ago 2017]. Disponível em: http://www.who.int/mediacentre/factsheets/fs2 97/en/.

10. Twycross R. Cuidados paliativos. Lisboa: Climepsi; 2000. 184 p.

11. Silva RC. As atividades como recurso para a pesquisa. Cad Ter Ocup UFSCar. 2013; 21(3):46170.

12. Boemer MR. Sobre cuidados paliativos. São Paulo: USP; 2009.

13. Palm RCM. Oncologia. In: Cavalcanti A, Galvão C. Terapia ocupacional: fundamentação e prática. Rio de Janeiro: Guanabara Koogan; 2007.

14. Luz JD, Lima JAS, Monteiro LG. Automedicação do idoso. [Trabalho de Conclusão de Curso]. Escola Superior de Saúde, Universidade do Mindelo, Mindelo, Cabo Verde; 2013.

15. Camacho ACLF, Santos RC, Joaquim FB, Louredo DS, Morais IS, Silva EA. Estudo comparativo sobre a capacidade funcional de pacientes adultos e idosos com úlceras venosas. Rev Pesqui Cuid Fundam. (Online) 2015 [citado em 23 jan 2017]; 7(1):1954-66. Disponível em: http://www.seer.unirio.br/index.php/cuidadofundam ental/article/viewFile/3505/pdf_1435.

16. Silva D, Lopes EL, Braga Junior SS. Pesquisa quantitativa: elementos, paradigmas e definições. Rev Gest Secr. 2014; 5(1):1-18.

17. Ferraz MB, Oliveira LM, Araujo PMP, Atra E, Tugwell P. Cross cultural reliability of the physical 
ability dimension of the Health Assessment Questionnaire. J Rheumatol. 1990; 17(6): 813-7. 18. Janssens X, Decuman S, Keyser F. Comparison of the belgian rheumatoid arthritis disability Assessment and Health Assessment Questionnaires as tools to predict the need for support measures in patients with rheumatoid arthritis. PLos ONE. 2016; 11(1):1-11.

19. Ebbevi D, Essen A, Forsberg HH. Persons with rheumatoid arthritis challenge there evince of the health assessment questionnaire: a qualitative study of patient perception. BMC Musculoskelet Disord. 2017; 18(189):1-10.

20. Santos EO. Caracterização molecular do Vírus Linfotrópico de Células T de Humano (HTLV) em pacientes com paraparesia espástica tropical / mielopatia (PET/MAH), portadores e gestantes em Alagoas. [Tese]. Recife: Universidade Federal de Pernambuco; 2013.

21. Oliveira EH, Silva FL, Silva ML. Perfil epidemiológico dos doadores de sangue infectados pelo vírus HTLV I/II, no Estado do Piauí. Rev Interdisciplin. 2015; 8(1):149-56.

22. Felix JC. Caracterização das variantes genotípicas do HTLV em um grupo de pacientes acompanhados em hospital universitário do estado do Rio de Janeiro. [Dissertação] Rio de Janeiro: Instituto Oswaldo Cruz; 2014. 77 f.
23. Coutinho IJ, Castro Filho BG, Lima J, Castello C, Eiter D, Grassi MFR. Impacto da mielopatia associada ao HTLV / paraparesia espástica tropical (TSP/HAM) nas atividades de vida diária (AVD) em pacientes infectados pelo HTLV-1. Acta Fisiátrica. 2011; 18(1):6-10.

24. Melo AGC, Caponero RO. Futuro em cuidados paliativos. In: Santos FS. Cuidados paliativos: diretrizes, humanização e alívio de sintomas. São Paulo: Atheneus; 2011.

25. Henning RCV. Terapia ocupacional nos cuidados paliativos oncológicos. Joinville: FGG; 2010.

26. Rocha EF, Mello MAF. Os sentidos do corpo e da intervenção hospitalar. In: Carlo MMRP, Luzo MCM. Terapia ocupacional: reabilitação física e contextos hospitalares. São Paulo: Roca; 2004.

CONTRIBUIÇÕES
Ádria Cecília Botelho da Rocha e Priscila
Sarmento de Almeida realizaram a pesquisa
de campo, análise e organização dos dados, e
redação. Luiz Fábio Magno Falcão, Denise
da Silva Pinto e Hellen Thaìs Fuzii
contribuíram na revisão do trabalho. Victor
Augusto Cavaleiro atuou na supervisão do
trabalho e redação.

\footnotetext{
Como citar este artigo (Vancouver)

Corrêa VAC, Rocha ACB, Almeida PS, Pinto DS, Falcão LFM, Fuzii HT. Capacidade funcional em indivíduos com paraparesia espástica tropical/mielopatia associada ao HTLV-1. REFACS [Internet]. 2018 [citado em inserir dia, mês e ano de acesso]; 6(1):7-14. Disponível em: inserir link de acesso. DOI: inserir link do DOI.

Como citar este artigo (ABNT)

CORRÊA, V. A. C. et al. Capacidade funcional em indivíduos com paraparesia espástica tropical / mielopatia associada ao HTLV-1. REFACS, Uberaba, v. 6, n. 1, p. 7-14, 2018. Disponível em: <inserir link de acesso >. Acesso em: inserir dia, mês e ano de acesso. DOI: inserir link do DOI.

\section{Como citar este artigo (APA)}

Corrêa, V. A. C., Rocha, A. C. B., Almeida, P. S., PINTO, D. S., Falcão, L. F. M. \& Fuzii, H. T. (2018). Capacidade funcional em indivíduos com paraparesia espástica tropical/mielopatia associada ao HTLV-1. REFACS, 6(1), 7-14. Recuperado em: inserir dia, mês e ano de acesso de inserir link de acesso. DOI: inserir link do DOI.
} 